\title{
Habilidade de localização e lateralização sonora em deficientes visuais
}

\section{Sound localization and lateralization in individuals with visual deficiency}

\author{
Thaiana Lice Lopes Dias ${ }^{1}$, Liliane Desgualdo Pereira ${ }^{2}$
}

\begin{abstract}
RESUMO
Objetivo: Verificar o desempenho de ouvintes, cegos ou com visão subnormal, nos testes de localização sonora em cinco direções e de lateralização, comparando-o com indivíduos videntes, com sensibilidade auditiva normal, nas mesmas tarefas. Métodos: Foram selecionados 21 indivíduos com sensibilidade auditiva normal, faixa etária média de 30 anos, de ambos os gêneros, reunidos pela presença cegueira e/ou visão subnormal congênita ou adquirida. Foram realizados os testes que compõem a avaliação audiológica básica, o Teste de Localização Sonora e o Teste de Lateralização com Som Verbal, nas técnicas ascendente e descendente. Os resultados obtidos com os testes realizados pelo grupo de 21 indivíduos com deficiência visual foram comparados com os de 40 adultos videntes e ouvintes normais. Resultados: No grupo estudo (deficientes visuais), tanto na técnica ascendente quanto na descendente, existem diferenças estatisticamente significantes entre os limiares de lateralização sonora para as orelhas direita e esquerda. O maior limiar de lateralização foi observado na orelha esquerda em cada grupo. Em cada técnica, ascendente e descendente, não existiram diferenças estatisticamente significantes para o limiar de lateralização por orelha, em cada grupo. Comparando os dados do teste de lateralização sonora do grupo de deficientes visuais por técnica e orelha, com os dados do grupo de comparação de videntes, verificamos que existiram diferenças estatisticamente significantes em ambas as técnicas e orelhas, com exceção da orelha esquerda, na técnica ascendente. Conclusão: Os deficientes visuais avaliados têm um excelente desempenho em lateralização de sons, muito melhor do que sujeitos videntes e ouvintes típicos ou normais.
\end{abstract}

Descritores: Audição; Testes auditivos; Localização de som; Cegueira; Portadores de deficiência visual

\section{INTRODUÇÃO}

A localização sonora é a habilidade de identificar o local de origem do som ${ }^{(1-2)}$. Esta habilidade é fundamental para o desenvolvimento da atenção seletiva e, portanto, auxilia na comunicação oral ${ }^{(2)}$.

Lateralização é o termo usado para o comportamento de localizar sons à direita e à esquerda, tendo como referência a cabeça do indivíduo. A localização intracraniana (sensação de localização de som dentro da cabeça) é denominada lateralização, em oposição à localização, que é percebida fora da cabeça, de acordo com a direção e distância da fonte sonora ${ }^{(3)}$.

Para a tarefa de localizar sons, os indivíduos necessitam

Trabalho de iniciação científica (PIBIC-CNPq) e de Bolsa de Produtividade (CNPq) realizado no Ambulatório da Disciplina de Distúrbios da Audição do Departamento de Fonoaudiologia da Universidade Federal de São Paulo - UNIFESP em parceria com a Fundação Dorina Nowill para Cegos - São Paulo (SP), Brasil.

(1) Pós-graduanda em Distúrbios da Comunicação Humana pela Universidade Federal de São Paulo - UNIFESP - São Paulo (SP), Brasil.

(2) Livre Docente, Professora Associada do Departamento de Fonoaudiologia da Universidade Federal de São Paulo - UNIFESP - São Paulo (SP), Brasil.

Endereço para correspondência: Thaiana Lice Lopes Dias. R. Botucatu, 802, Vila Clementino, São Paulo - SP, CEP 04023-062. E-mail: thaiana@terapiadafala.com.br

Recebido em: 26/11/2007; Aceito em: 5/9/2008 avaliar comparativamente o nível de intensidade do som que chegou a cada orelha, o tempo de chegada do som a cada orelha, bem como a fase de chegada do som a cada orelha. Toda essa análise é realizada sem a consciência do indivíduo. Trata-se de um comportamento que envolve o funcionamento eficiente das vias auditivas do sistema nervoso central e do córtex, além de uma adequada sensibilidade auditiva em ambas as orelhas do indivíduo. Utilizando-se uma gravação de sons e apresentação desses sons, um em cada orelha, pode-se isolar algumas das variáveis que o indivíduo usa para lateralizar o som. O desenvolvimento do comportamento de localização sonora auxilia o indivíduo a separar sons do meio ambiente, analisar acusticamente características de freqüência, intensidade e duração, importantes no reconhecimento de fala.

A cegueira é definida como "acuidade visual corrigida 20/200 ou menos no olho melhor ou um campo visual de não mais que 20 graus no olho menos afetado"(4). Pode-se ainda falar em "ambliopia e amaurose que são os termos usados para a perda parcial e completa da visão, respectivamente, de um ou ambos os olhos, na ausência de sinais oftalmológicos ou objetivos marcantes"(5).

É importante lembrar que déficits visuais comprometem a segurança para a realização das atividades de vida diária (AVD).

Neste trabalho, foi utilizado o Teste de Lateralização com Som $\operatorname{Verbal}^{(6)}$, que mede a localização de sons à orelha direita 
e à orelha esquerda, por meio da capacidade de identificar aquela orelha que recebeu primeiro no tempo o estímulo verbal. Esse teste foi estudado em ouvintes adultos normais ${ }^{(6)}$, e em ouvintes com e sem educação musical. O comportamento de localização sonora em indivíduos cegos pode mostrar a sua capacidade de separar informações acústicas e prognosticar o desempenho dos mesmos na comunicação oral.

Sabe-se que indivíduos cegos não recebem maior quantidade de estímulos auditivos que os indivíduos videntes ${ }^{(7)}$. $\mathrm{O}$ desenvolvimento de suas capacidades sensoriais ocorreria com base na utilização, cada vez melhor, de seus sentidos remanescentes ${ }^{(8)}$. Podemos, então, afirmar que habilidades auditivas, como a resolução temporal, seriam desenvolvidas pelo déficit sensorial (visual).

Acredita-se que o estudo do comportamento auditivo de localização sonora, em indivíduos deficientes visuais, pode mostrar a sua capacidade de lidar com informações acústicas dicóticas e, ainda, prognosticar o desempenho dos mesmos na comunicação auditivo-verbal e na realização das atividades de vida diária (AVD). Além disso, sabemos que tal população é pouco investigada no meio científico. Desta forma, faz-se necessária a realização de pesquisas nesta área.

A hipótese deste trabalho é de que sujeitos deficientes visuais têm melhor desempenho em tarefas de processamento auditivo, tais como: localização e lateralização sonora, já que um indivíduo cego adquire conhecimentos por meio de experiências que não incluem a visão. Isto é, todo o seu aprendizado procede por outros caminhos ${ }^{(9)}$.

Esperamos com este estudo, compreender os aspectos teóricos e teórico-práticos do processamento auditivo de sujeitos cegos e, desta forma, ampliar os conhecimentos sobre a audição desta população tão pouco investigada cientificamente pela Fonoaudiologia.

O objetivo dessa pesquisa é verificar o desempenho de ouvintes jovens e adultos cegos ou com visão subnormal, no teste de lateralização sonora.

\section{MÉTODOS}

O trabalho foi analisado e aprovado pelo comitê de ética em pesquisas da Universidade Federal de São Paulo/ Hospital São Paulo (CEP 1078/04).

Foram avaliados 24 indivíduos com deficiência visual. Destes apenas três apresentaram limiares auditivos rebaixados e foram, portanto, excluídos da amostra, após serem devidamente orientados sobre a deficiência auditiva observada. Dos 21 indivíduos, todos passaram no teste de localização sonora, e foram assim incluídos na amostra.

Os critérios de inclusão para o grupo com diagnóstico de deficiência visual foram: função auditiva periférica normal (limiares de audibilidade até $25 \mathrm{~dB}$ NA nas freqüências de 500 , 1000 e 2000 Hz, sem gap aéreo-ósseo; timpanometria com curva do tipo A, bilateralmente, e reflexos acústicos estapedianos presentes nas frequiências de 500, 1000 e $2000 \mathrm{~Hz}$; passar no teste de localização sonora em cinco direções ${ }^{(10)}$; ausência de evidências de problemas neurológicos ou cognitivos. $\mathrm{O}$ grupo de indivíduos deficientes visuais, denominado grupo estudo, foi selecionado entre os membros da Fundação Dorina Nowill para cegos, que concordaram voluntariamente em participar desse trabalho, após terem sido devidamente esclarecidos.

Cada indivíduo foi submetido a um levantamento da história clínica, no qual foram obtidos dados de identificação do grupo estudo (nome, idade, sexo, profissão) e informações referentes a queixas de dificuldade de aprendizagem, memória e compreensão em ambientes ruidosos.

Os resultados obtidos com os testes realizados pelo grupo de indivíduos com deficiência visual (grupo estudo) foram analisados, utilizando o grupo $(\mathrm{n}=40)$ avaliado pela autora de outro estudo ${ }^{(6)}$, que caracterizou a lateralização sonora em adultos do sexo feminino, faixa etária entre 18 e 25 anos, ouvintes e videntes (grupo comparação).

Todos os testes auditivos foram realizados no Ambulatório da Disciplina de Distúrbios da Audição - Departamento de Fonoaudiologia da Universidade Federal de São Paulo (UNIFESP). Inicialmente, foi realizada a avaliação audiológica básica: audiometria tonal liminar - realizada em audiômetro modelo AC33, de marca Interacoustics, calibrado segundo a norma ANSI 69; audiometria vocal: limiar de reconhecimento de fala (SRT) e Índice percentual de Reconhecimento de fala (IPRF); medidas de imitância acústica: timpanometria e pesquisa dos reflexos acústicos contralaterais, realizada em imitanciômetro modelo AZ7, de marca Interacoustics.

O processamento auditivo envolvido no comportamento de localização sonora foi avaliado por meio de dois testes realizados em cabina acusticamente tratada: Teste de Localização Sonora (TLS) e Teste de Lateralização com Som Verbal (TLSV).

No TLS, o sujeito foi instruído a indicar o local de origem do som (acima, direita, esquerda, frente, trás) produzido por um objeto sonoro (guizo 5) com faixa de freqüências ampla e nível de intensidade maior nas frequiências altas. Com esse procedimento, procurou-se buscar informações sobre a interação binaural ${ }^{(3)}$.

No TLSV, o indivíduo foi instruído a apontar o local imaginário da fonte sonora. A resposta de localização é medida em milissegundos (ms). Esse comportamento foi denominado de resolução temporal binaural ${ }^{(6)}$. Àqueles sons apresentados simultaneamente em ambas as orelhas, isto é, sem diferença de tempo de chegada em cada orelha, provocam uma sensação de som (fonte sonora imaginária) no meio da cabeça e à identificação desta fonte sonora imaginária no centro da cabeça, dá-se o nome de centralização. À identificação da fonte sonora imaginária correspondente à sensação do som do lado em que ele foi apresentado primeiro, dá-se o nome de lateralização à direita ou à esquerda. A palavra "paca" foi o estímulo utilizado, gravado em disco a laser, apresentado de maneira binaural, com tempo de atraso interaural entre $0 \mathrm{e}$ $500 \mathrm{~ms}$. O teste foi realizado em cabina acústica, utilizandose Compact Disc Sony ${ }^{\circledR}$, modelo D-802K e fones de ouvido calibrados por um engenheiro acústico. As respostas foram registradas em um protocolo de registro de dados, elaborado para o atual trabalho.

O nível de significância para a análise estatística adotado foi de 0,05 ou $5 \%$. Os intervalos de confiança construídos ao longo do trabalho foram construídos com $95 \%$ de confiança. Foi utilizado o Teste de Igualdade de Duas Proporções para 
comparar a proporção de homens e mulheres. As orelhas: direita e esquerda foram comparadas em cada uma das técnicas por meio do teste de igualdade de duas médias, também chamado de teste $\mathrm{Z}$.

\section{RESULTADOS}

Foram selecionados 21 indivíduos com sensibilidade auditiva normal, na faixa etária variando de 20 a 42 anos (média de 30 anos), de ambos os gêneros (42,9\% do sexo masculino e 57,1 do sexo feminino, $\mathrm{p}=0,355$ ), reunidos pela presença cegueira e/ou visão subnormal congênita ou adquirida.

No grupo estudo, foi observado que os limiares de lateralização médios para a orelha esquerda $(\mathrm{TA}=132,62$ e $\mathrm{TD}=144,05)$ são maiores do que para a orelha direita $(\mathrm{TA}=89,52$ e $\mathrm{TD}=102,62)$, tanto na técnica ascendente (TA) quanto na descendente (TD).

Ao compararmos os limiares por orelha direita ou esquerda entre as técnicas: ascendente ou descendente do teste, não houve diferenças, isto é, o desempenho por orelha ( $\mathrm{p}$-valores $\mathrm{OD}=0,468$ e $\mathrm{OE}=0,582$ ) nas técnicas descendente e ascendente foi considerado semelhante.

Ao comparar os dados do teste de Lateralização Sonora, aplicado em deficientes visuais por técnica e orelha com o grupo de comparação de videntes, verificamos que existiram diferenças estatisticamente significantes em ambas as técnicas e orelhas $(\mathrm{p}<0,001)$, com exceção a orelha esquerda, na técnica ascendente $(\mathrm{p}=0,336)$.

Mostramos na Tabela 1 as medidas descritivas calculadas para os limiares de lateralização para as orelhas: direita e esquerda, nas técnicas ascendente e descendente.

No grupo estudo (deficientes visuais), verificamos que a melhor lateralização sonora foi observada para a orelha direita, independente da técnica utilizada. Ao contrário, no grupo comparação (videntes), verificou-se que a melhor lateralização sonora ocorreu na orelha esquerda, independente da técnica utilizada, ascendente ou descendente (Figura 1).

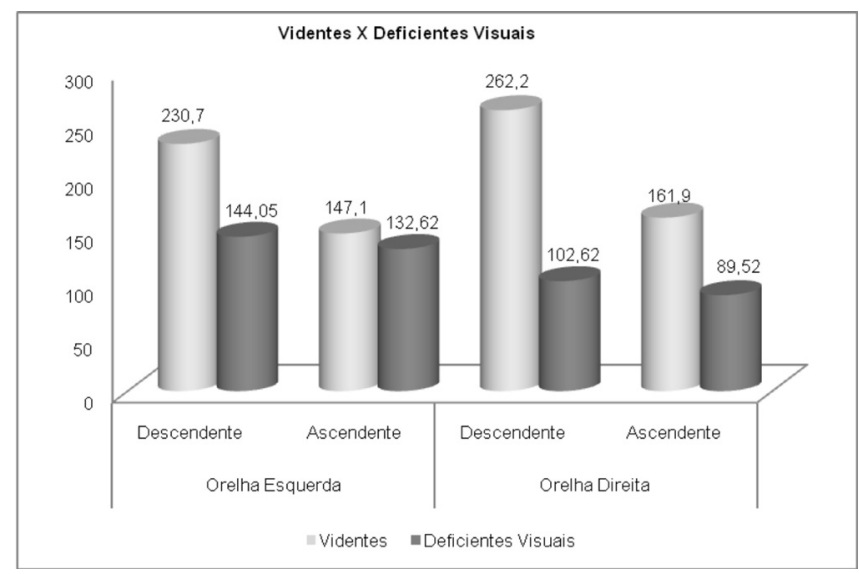

Figura 1. Limiares de lateralização para orelhas direita e esquerda nas técnicas ascendente e descendente dos grupos comparação (videntes) e estudo (deficiente visuais)

Verificamos que a habilidade de lateralização sonora, com o mesmo estímulo verbal apresentado em ambas as orelhas, com diferentes tempos (em microssegundos) de chegada em cada uma, avaliada em deficientes visuais com mecanismos fisiológicos normais de detecção de tons puros (sensibilidade auditiva) e de discriminação da direção da fonte sonora (localização), foi melhor quando comparada a de videntes ouvintes normais.

Tabela 1. Medidas descritivas dos limiares de lateralização para as orelhas direita e esquerda nas técnicas ascendente e descendente para comparação, considerando variável orelha e técnica no grupo estudo (deficientes visuais)

\begin{tabular}{|c|c|c|c|c|}
\hline \multirow[t]{2}{*}{ Medidas descritivas } & \multicolumn{2}{|c|}{ Descendente } & \multicolumn{2}{|c|}{ Ascendente } \\
\hline & OE & OD & OE & OD \\
\hline Média & 144,05 & 102,62 & 132,62 & 89,52 \\
\hline Mediana & 113 & 91 & 113 & 91 \\
\hline Desvio Padrão & 72,02 & 58,43 & 62,21 & 58,42 \\
\hline Mínimo & 45 & 23 & 23 & 23 \\
\hline Máximo & 304 & 227 & 272 & 272 \\
\hline População (n) & 21 & 21 & 21 & 21 \\
\hline Limite inferior & 113,24 & 77,63 & 106,01 & 64,54 \\
\hline Limite superior & 174,85 & 127,61 & 159,23 & 114,51 \\
\hline \multirow[t]{2}{*}{ p-valor (OD X OE) } & \multicolumn{2}{|c|}{ Técnica descendente } & \multicolumn{2}{|c|}{ Técnica ascendente } \\
\hline & \multicolumn{2}{|c|}{$0,041^{*}$} & \multicolumn{2}{|c|}{$0,021^{*}$} \\
\hline $\mathrm{p}$-valor (ascendente $\mathrm{X}$ & \multicolumn{2}{|c|}{ OD } & \multicolumn{2}{|c|}{ OE } \\
\hline descendentes) & \multicolumn{2}{|c|}{0,468} & \multicolumn{2}{|c|}{0,582} \\
\hline \multirow[t]{3}{*}{$\mathrm{p}$-valor (videntes $\mathrm{X}$ deficientes visuais) } & & \multicolumn{2}{|c|}{ Técnica descendente } & Técnica ascendente \\
\hline & \multicolumn{2}{|c|}{ OD } & \multicolumn{2}{|c|}{$<0,001^{*}$} \\
\hline & \multicolumn{2}{|c|}{$\mathrm{OE}$} & \multicolumn{2}{|c|}{0,336} \\
\hline
\end{tabular}

Legenda: * $\mathrm{p}$-valores considerados estatisticamente significativos perante o nível de significância adotado; OE = orelha esquerda; OD = orelha direita 


\section{DISCUSSÃO}

Os achados deste estudo demonstram que existe uma percepção auditiva diferenciada nos indivíduos deficientes visuais. Resultados semelhantes foram descritos por outros autores $^{(8,11-15)}$.

$\mathrm{Na}$ atenção direcionada para a audição periférica espacial, a localização de sons mostra-se significativamente melhor nos indivíduos cegos do que nos videntes, bem como nas tarefas de localização sonora monoaural ${ }^{(11)}$.

A visão é responsável pela integração das atividades motora, perceptiva e mental ${ }^{(16)}$. A experienciação visual é utilizada para a correção da localização sonora entre as coordenadas verticais do campo da audição e o corpo sem, no entanto, ser de grande exatidão na audição espacial ${ }^{(11)}$.

O fenômeno da plasticidade de modalidade cruzada (crossmodal plasticity) foi registrado em indivíduos cegos que foram submetidos a estímulos auditivos ${ }^{(12)}$ e táteis ${ }^{(17)}$. Ocorreu processamento desses estímulos não visuais no córtex visual.

Imagens de ressonância magnética funcional de indivíduos com privação sensorial demonstram que a plasticidade neuronal contribui para um melhor desempenho de indivíduos deficientes visuais, em atividades como processamento de fala ${ }^{(13)}$.

A plasticidade neural é a "capacidade que o sistema nervoso central possui em modificar algumas das suas propriedades morfológicas e funcionais, em resposta às alterações do ambiente"(18). Estes mesmos autores descrevem diversos fatores que estão relacionados com a plasticidade como característicos da lesão, idade do paciente, estado emocional, cognição e ambiente terapêutico.

Indivíduos deficientes visuais tiveram melhor desempenho em tarefas de discriminação de sons verbais (consoante-vogal) em escuta dicótica, quando comparados com indivíduos videntes, o que indica que houve reorganização hemisférica nos indivíduos cegos, em ambos os níveis de processamento de informações (sensorial e cognitiva), na modalidade sensorial auditiva $^{(14)}$

Quanto ao comportamento dos indivíduos deficientes visuais na realização dos testes, pudemos observar maior concentração aos estímulos auditivos, o que poderia justificar o melhor desempenho no teste de lateralização sonora.

O teste de lateralização sonora pode ser considerado não só como um instrumento de avaliação, mas também como um material para treinamento auditivo. Diversos estudos com treinamento auditivo demonstram bons resultados no desenvolvimento de habilidades auditivas ${ }^{(19-21)}$.

Sabemos que habilidades auditivas como o processamento temporal são de extrema importância para a identificação de elementos fonéticos, contribuindo assim para a percepção de fala ${ }^{(22-23)}$. Podemos, então, afirmar que a percepção de fala de deficientes visuais é melhor do que a de indivíduos videntes, pelo fato de processarem melhor e com maior efetividade as propriedades físicas dos sons da fala (freqüência, intensidade e duração) $)^{(13-15)}$.

O que poderia ter proporcionado essa melhor percepção auditiva? Para responder a essa pergunta, fomos conhecer alguns programas de reabilitação de instituições especializadas em deficientes visuais. Na Fundação Dorina Nowill para cegos, por exemplo, há programas de reabilitação para todas as faixas etárias. Os bebês são estimulados precocemente e as famílias orientadas a proporcionar ambiente favorável para um bom desenvolvimento psicomotor. A criança préescolar é estimulada nas habilidades psicomotoras, requisitadas posteriormente, na fase escolar. Nesta fase, inicia-se a educação especial e treinamento de mobilidade e orientação, AVD, aprendizagem de códigos Braile especiais e orientação psicológica. O jovem, adulto e idoso deficiente visual passam pelo programa com o objetivo de reintegração no lar, escola, trabalho e comunidade ${ }^{(24)}$.

Os dados obtidos neste trabalho nos faz supor que o treinamento auditivo com o uso de propriedades físicas dos sons da fala poderia ser utilizado para melhorar o reconhecimento de fala, tanto em indivíduos jovens, ainda em desenvolvimento, quanto em idosos que, pelo próprio processo de envelhecimento, sofrem perdas sensoriais, incluindo visuais e auditivas ${ }^{(25-28)}$.

Muitos programas de reabilitação para deficientes visuais incluem treinamento de equilíbrio, que poderia também ser utilizado com a população idosa. É importante lembrar que déficits visuais comprometem a segurança para a realização das atividades de vida diária (AVD). Déficits visuais e/ou auditivos são preditores de quedas em idosos ${ }^{(29)}$.

Desta forma, a reabilitação auditiva baseada na integração sensorial e motora poderia contribuir para o aprendizado perceptual envolvido na competência comunicativa e lingüística. Com este trabalho, acredita-se poder recomendar que o desenvolvimento das habilidades auditivas em processo terapêutico fonoaudiológico deva ser baseado em estratégias que utilizem essa abordagem integrada.

A Fonoaudiologia tem muito que aprender e aprimorar com a experiência dos indivíduos deficientes visuais. Programas de treinamento auditivo podem ser reformulados, considerando-se a prática das atividades cotidianas vividas por indivíduos com privação sensorial. Ainda há muito que estudar.

O treinamento auditivo, embora não formal, é muito utilizado na reabilitação de deficientes visuais (ex: sons ambientais, batidas da bengala em diferentes pisos). Conhecer esses programas de reabilitação especializada e somar os benefícios do treinamento formal acrescentaria maior segurança para a realização das atividades de vida diária, bem como melhor qualidade de vida.

\section{CONCLUSÃO}

Os deficientes visuais tiveram um melhor desempenho na lateralização de sons, quando comparados a sujeitos videntes e ouvintes típicos ou normais.

\section{AGRADECIMENTOS}

Agradecemos ao CNPq e aos funcionários e voluntários da Fundação Dorina Nowill para Cegos pela imensa colaboração. 


\begin{abstract}
Purpose: To verify the auditory performance of blind or subnormal sight individuals in tests for sound localization in five directions and lateralization, compared to individuals with normal sight and normal auditory sensibility. Methods: Twenty-one individuals with normal auditory sensibility were selected, mean age of 30 years, both genders, grouped according to presence of blindness and/or acquired or congenital subnormal sight. Audiological evaluation tests, Sound Localization Test and Lateralization Test with verbal sound, using both ascending and descending techniques, were carried out. Results obtained on the tests by the 21 subjects with visual deficiency were compared to those of 40 adults with normal sight and hearing. Results: The visually impaired group, both in ascending and descending techniques, presented statistically significant differences between sound lateralization limits for right and left ears. The higher lateralization threshold was observed on the left ear in each group. In each technique, ascending and descending, there were no statistically significant differences in the lateralization threshold per ear in each group. Comparing data from the sound lateralization test of the visually impaired per technique and ear, with data from the comparison group with normal sight, it was verified that there were statistically significant differences for both techniques and ears, except for the left ear in the ascending technique. Conclusion: The visually impaired evaluated showed an excellent performance in sound lateralization, much better than individuals with normal sight and hearing.
\end{abstract}

Keywords: Hearing; Auditory tests; Sound localization; Blindness; Visually impaired persons

\title{
REFERÊNCIAS
}

1. Boothroyd A. Speech acoustics and perception. Austin (Texas): Pro-Ed; 1986.

2. Pereira LD e Cavadas M. Processamento auditivo central. In: Frota S. Fundamentos em fonoaudiologia - Audiologia. Rio de Janeiro: Guanabara Koogan; 1998. p.135-46.

3. Pereira LD, Schochat E. Processamento auditivo central - Manual de avaliação. São Paulo: Lovise; 1997.

4. Vaughan D, Asbury T. Oftalmologia geral. São Paulo: Atheneu; 1977.

5. Miller SJ. Enfermidades dos olhos de Parson. 16a ed. São Paulo: Artes Médicas; 1981.

6. Tedesco MLF. Lateralização sonora: diferença interaural mínima de tempo em ouvintes normais [tese]. São Paulo: Universidade Federal de São Paulo; 2002.

7. Elbert T, Sterr A, Rockstroh B, Pantev C, Müller MM, Taub E. Expansion of the tonotopic area in the auditory cortex of the blind. J Neurosci. 2002;22(22):9941-4.

8. Ballesteros S, Millar S, Reales JM. Symmetry in haptic and in visual shape perception. Percept Psychophys. 1998;60(3):389-404.

9. Martín MB. Reflexiones en torno a la educación de los deficientes visuales. Revista de Educación "Puerto Nueva" [Internet]. 1991 Mayo [cited 2007 Jun 22]; (13). Available from:: http://www.juntadeandalucia. es/averroes/caidv/interedvisual/ftp_p_/reflexiones_mbm_pn13.pdf

10. Pereira LD. Avaliação do processamento auditivo central. In: Lopes Filho O, organizador. Tratado de fonoaudiologia. São Paulo: Roca; 1997. (Houve uma troca no local marcado em pink- já corrigi!)

11. Lessard N, Paré M, Lepore F, Lassonde M. Early-blind human subjects localize sound sources better than sighted subjects. Nature. 1998;395(6699):278-80.

12. Kujala T, Alho K, Paavilainen P, Summala H, Näätänen R. Neural plasticity in processing of sound location by the early blind: an event-related potential study. Electroencephalogr Clin Neurophysiol. 1992;84(5):469-72.

13. Röder B, Stock O, Bien S, Neville H, Rösler F. Speech processing activates visual cortex in congenitally blind humans. Eur J Neurosci. 2002;16(5):930-6.

14. Hugdahl K, Ek M, Takio F, Rintee T, Tuomainen J, Haarala C, et al. Blind individuals show enhanced perceptual and attentional sensitivity for identification of speech sounds. Brain Res Cogn Brain Res. 2004;19(1):28-32. Erratum in: Brain Res Cogn Brain Res. 2004;20(2):328.

15. Stevens AA, Weaver K. Auditory perceptual consolidation in early-onset blindness. Neuropsychologia. 2005;43(13):1901-10.
16. Masini EF. A educação do portador de deficiência visual: as perspectivas do vidente e do não vidente. Em Aberto [Internet] 1993 Out/Dez [cited 2008 Nov 5]; 13(60):61-76. Disponível em: http://www.emaberto.inep. gov.br/index.php/emaberto/article/viewFile/888/795

17. Rösler F, Röder B, Heil M, Hennighausen E. Topographic differences of slow event-related brain potentials in blind and sighted adult human subjects during haptic mental rotation. Brain Res Cogn Brain Res. 1993;1(3):145-59.

18. Oliveira CEN, Salina ME. Annunciato NF. Fatores ambientais que influenciam a plasticidade do SNC. Acta Fisiátrica. 2001;8(1):6-13.

19. Gil D, Almeida CC, Phee AM, Artoni AL, Pellogia CC, Antunes F, et al. Efeito do treinamento auditivo para a percepção musical nos testes de padrão de freqüência e duração. Acta AWHO. 2000;19(2):64-7.

20. Schochat E, Carvalho LZ, Megale RL. Treinamento auditivo: avaliação da manutenção das habilidades. Pró-Fono. 2002; 14(1):93-98.

21. Ishii C, Arashiro PM, Pereira LD. Ordenação e resolução temporal em cantores profissionais e amadores afinados e desafinados. Pró-Fono. 2006;18(3):285-92.

22. Au A, Lovegrove B. Temporal processing ability in above average and average readers. Percept Psychophys. 2001;63(1):148-55.

23. Shinn JB. Temporal processing: the basics. Hear J. 2003;56(7):52.

24. Fundação Dorina Nowill para Cegos. Atendimento especializado [Internet]. São Paulo: Fundação Dorina Nowill para Cegos; c2003. [citado 2007 Set 9]. Disponível em: http://www.fundacaodorina.org.br/ fundacao/deficiencia.asp; 2007.

25. Quintero SM, Marotta RM, Marone SA. Avaliação do processamento auditivo de indivíduos idosos com e sem presbiacusia por meio do teste de reconhecimento de dissílabos em tarefa dicótica - ssw. Rev Bras Otorrinolaringol. 2002;68(1):28-33.

26. Babkoff H, Muchnik C, Ben-David N, Furst M, Even-Zohar S, Hildesheimer M. Mapping lateralization of click trains in younger and older populations. Hear Res. 2002;165(1-2):117-27.

27. Neves VT, Feitosa MA. Controvérsias ou complexidade na relação entre processamento temporal auditivo e envelhecimento? Rev Bras Otorrinolaringol. 2003;69(2):242-9.

28. Phillips SL, Gordon-Salant S, Fitzgibbons PJ, Yeni-Komshian G. Frequency and temporal resolution in elderly listeners with good and poor word recognition. J Speech Lang Hear Res. 2000;43(1):217-28.

29. Feder G, Cryer C, Donovan S, Carter Y. Guidelines for the prevention of falls in people over 65. The Guidelines' Development Group. BMJ. 2000;321(7267):1007-11. 